\title{
Femoral and tibial torsion measurements based on EOS imaging compared to 3D CT reconstruction measurements
}

\author{
Wenqiang Yan $^{1 \#}$, Xingquan Xu ${ }^{1 \#}$, Qian Xu ${ }^{1}$, Wenjin Yan $^{1}$, Ziying Sun ${ }^{1}$, Qing Jiang ${ }^{1,2}$, Dongquan Shi ${ }^{1}$ \\ ${ }^{1}$ State Key Laboratory of Pharmaceutical Biotechnology, Department of Sports Medicine and Adult Reconstructive Surgery, Nanjing Drum Tower \\ Hospital, The Affiliated Hospital of Nanjing University Medical School, Nanjing 210008, China; ${ }^{2}$ Laboratory for Bone and Joint Disease, Model \\ Animal Research Center (MARC), Nanjing University, Nanjing 210093, China \\ Contributions: (I) Conception and design: W Yan, X Xu, Q Jiang, D Shi; (II) Administrative support: None; (III) Provision of study materials \\ or patients: Q Xu, Z Sun; (IV) Collection and assembly of data: W Yan, X Xu, W Yan; (V) Data analysis and interpretation: W Yan, X Xu; (VI) \\ Manuscript writing: All authors; (VII) Final approval of manuscript: All authors. \\ \#These authors contributed equally to this work. \\ Correspondence to: Qing Jiang, MD, PhD; Dongquan Shi, MD, PhD. State Key Laboratory of Pharmaceutical Biotechnology, Department of Sports \\ Medicine and Adult Reconstructive Surgery, Nanjing Drum Tower Hospital, The Affiliated Hospital of Nanjing University Medical School, 321 \\ Zhongshan Road, Nanjing 210008, China. Email: qingj@nju.edu.cn; shidongquan1215@163.com.
}

Background: The EOS imaging system is an advanced piece of equipment for full-body imaging, but its reliability and reproducibility should be further verified.

Methods: A prospective study was conducted including 18 adult volunteers (36 lower extremities) ( $24 \pm 2$ years old). Femoral and tibial torsion were measured by both EOS imaging and three-dimensional computed tomography (3D CT) reconstruction. Bland-Altman plots were performed to evaluate the difference between femoral and tibial torsion measurements obtained by these two methods. The intraclass correlation coefficient (ICC) was used to evaluate intrareader agreement.

Results: The mean difference between the two methods was $3^{\circ}$ (range, $-9^{\circ}$ to $4^{\circ}$ ) for femoral torsion, $0^{\circ}$ (range, $-6^{\circ}$ to $6^{\circ}$ ) for tibial torsion and $0^{\circ}$ (range, $-4^{\circ}$ to $5^{\circ}$ ) for femorotibial torsion. No statistically significant difference between the measurements of the two methods was detected by Bland-Altman plots. With the exception of one measurement of femoral torsion, one measurement of tibial torsion and one measurement of femorotibial torsion, all EOS imaging measurements were within the $95 \%$ limits of agreement (the mean $\pm 1.96 \mathrm{SD})$. Intrareader agreement was statistically significant $(\mathrm{P}<0.001)$ for all measurements, with high ICCs. For EOS imaging, the ICC was 0.92 for the femoral measurement, 0.92 for the tibial measurement and 0.918 for the femorotibial measurement; the corresponding values for CT were $0.950,0.927$ and 0.889 .

Conclusions: There was good agreement between EOS imaging based and 3D CT reconstruction based technique in measuring femoral, tibial and femorotibial torsion; and good reliability and reproducibility of EOS Imaging in measuring femoral, tibial and femorotibial torsion was also verified.

Keywords: EOS imaging; three dimensional computed tomography reconstruction (3D CT reconstruction); femoral torsion; tibial torsion; femorotibial torsion

Submitted Apr 02, 2019. Accepted for publication Jul 24, 2019.

doi: $10.21037 /$ atm.2019.08.49

View this article at: http://dx.doi.org/10.21037/atm.2019.08.49 


\section{Introduction}

Femoral and tibial torsion are important for orthopedic surgeons to diagnose and treat patients of all age groups with lower-extremity problems, such as the torsional tibial deformities and idiopathic torsion disease. Precise measurement of the femoral and tibial torsion angle is required in preoperative planning of rotational osteotomies and arthroplasties, as well as many other lower limb operations (1-4).

Although two-dimensional computed tomography (CT) scanning is widely considered to be a relatively precise method for the quantitative analysis of femoral and tibial torsion angle, several different measurement techniques have been proposed $(5,6)$. The reliability and reproducibility of two-dimensional CT scans are limited by anatomical determination, especially along the femoral neck axis $(7,8)$, and by positional variables that influence the accuracy of measurement (9). 3D CT reconstruction enables the precise determination of femoral and tibial torsion without influence from femoral or tibial positioning and the determination of femoral neck axis $(10,11)$.

The EOS system is an advanced piece of equipment for full-body imaging $(12,13)$. Two perpendicular plane projections are generated simultaneously through a single scan. Generic models of the femur, tibia, and fibula can be altered with the use of dedicated software (sterEOS, France), thereby generating a three-dimensional model of the lower extremities. With 3D model reconstruction, many clinical parameters, including femoral and tibial torsion, can be computed automatically (14).

To the best of our knowledge, there was little study on the comparison between EOS Imaging based and 3D CT reconstruction based technique in measuring femoral, tibial and femorotibial torsion $(15,16)$. Therefore, the purpose of this study was to investigate: (I) whether there was good agreement between EOS imaging based and 3D CT reconstruction based technique in measuring femoral, tibial and femorotibial torsion; (II) to verify the repeatability of the EOS Image reading in measuring femoral, tibial and femorotibial torsion.

\section{Methods}

\section{Population}

After approval from the institutional ethics committee and after obtaining informed consent, 18 healthy volunteers (14 males, 4 females; average age: 24 years, age range:
22-27 years) were enrolled. No visible abnormalities of the lower limbs or pelvis were observed in these volunteers.

\section{Radiographic acquisition and measurement protocol}

EOS imaging and measurement protocol: biplanar X-rays were completed by an EOS device (EOS Imaging, France). Simultaneous anteroposterior and lateral images $(80 \mathrm{kV}$, tube voltage; $200 \mathrm{~mA}$, tube current) were acquired by this system, with the patient in an upright, physiological loadbearing position. Then, for better identification of the femoral condyle, tibial plateau and malleoli on the lateral radiograph, the volunteers were instructed to place one foot (usually the left) slightly anterior to the other (shiftedfeet standing position) (Figure 1). The obtained radiology parameters were automatically estimated by the imaging system. The 3D modeling and angle measurement of the lower limb were completed by the sterEOS software. The defined lines are projected on a plane orthogonal to the mechanical axis of the femur or tibia to the angle between them is measured (Figure 2). The defined lines included the femoral neck axis, the femoral posterior bicondylar axis, the tibial posterior bicondylar axis and the transmalleolar axis. The femoral neck axis and the axis connecting the most posterior point on each femoral condyle are projected on a plane that is orthogonal to the femoral mechanical axis. The femoral torsion is defined as the acute angle between the two projected lines. If the measured angle is positive, then the femoral neck is considered to be anteverted. The tangential axis at the back of the tibial segment and the transmalleolar axis are projected on a plane that is orthogonal to the tibial mechanical axis. The tibial torsion is defined as the acute angle between the two projected lines. If the measured angle is positive, then the malleolus is considered to rotate externally relative to the tibia. The axis connecting the most posterior point on each femoral condyle and the tangential axis at the back of the tibial segment are projected on a plane that is orthogonal to the femoral mechanical axis. The femorotibial torsion is defined as the acute angle between the two projected lines. If the measured angle is positive, then the tibia is considered to rotate externally relative to the femur.

The sterEOS software uses a technique based on threedimensional parametric models and statistical inferences. The reconstruction procedure is a software based stepby-step operation including the recognition of anatomical landmarks (Figure 3). Three-dimensional models are semiautomatically adjusted to the bony contour of the 

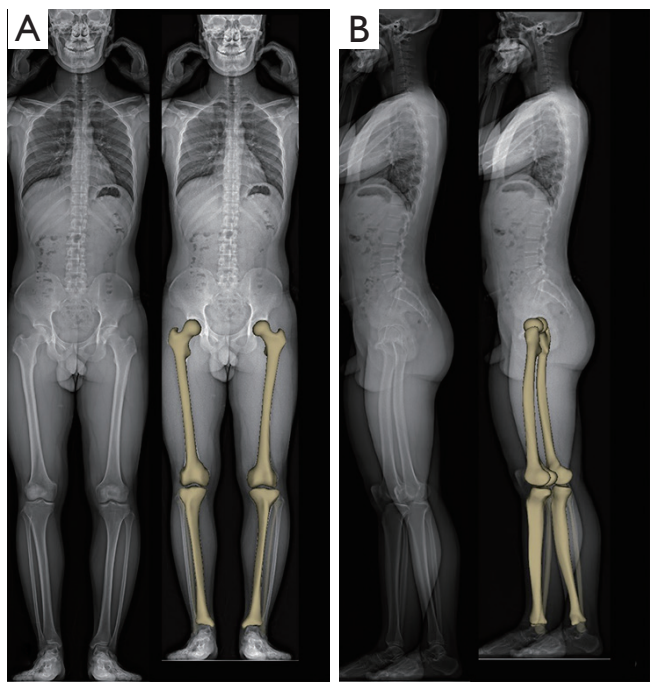

Figure 1 Three-dimensional model based on EOS imaging system. (A and B) Anteroposterior and lateral radiographs (left) are acquired for $3 \mathrm{D}$ reconstruction. Fitting model of lower extremity is adapted to osseous contour on radiographs (right). EOS, EOS imaging system (EOS imaging). lower limb. The patient's lower limb was represented by the adapted 3D model (Figure 1) that was generated by the fitting process. The discernment of anatomical landmarks is critical for accurate measurements. These marks include the greater trochanter, posterior contour of the femoral condyle, posterior contour of the medial and lateral sides of the tibial plateau, and the malleoli (Figure 3).

$\mathrm{CT}$ reconstruction and measurement protocol: The volunteers were scanned by a CT scanner (GE Discovery CT $750 \mathrm{HD}$, GE Medical Systems) in the supine position with hips extended and thighs horizontal and parallel. Axial images $(120 \mathrm{kV}$, tube voltage; $185 \mathrm{mAs} / \mathrm{slice}$, tube current; 0.426 , pitch factor; $512 \times 512$, matrix; $0.625 \mathrm{~mm}$, reconstruction thickness) of the hip, knee, and ankle were obtained without body movement.

The images were reconstructed at a $3 \mathrm{D}$ reconstruction workstation (Mimics Research 17.0) to produce 3D models (Figure $4 A$ ). After reconstruction of the proximal and distal femur, the $3 \mathrm{D}$ model of the proximal and bilateral condyles of the femur were aligned to form one image.
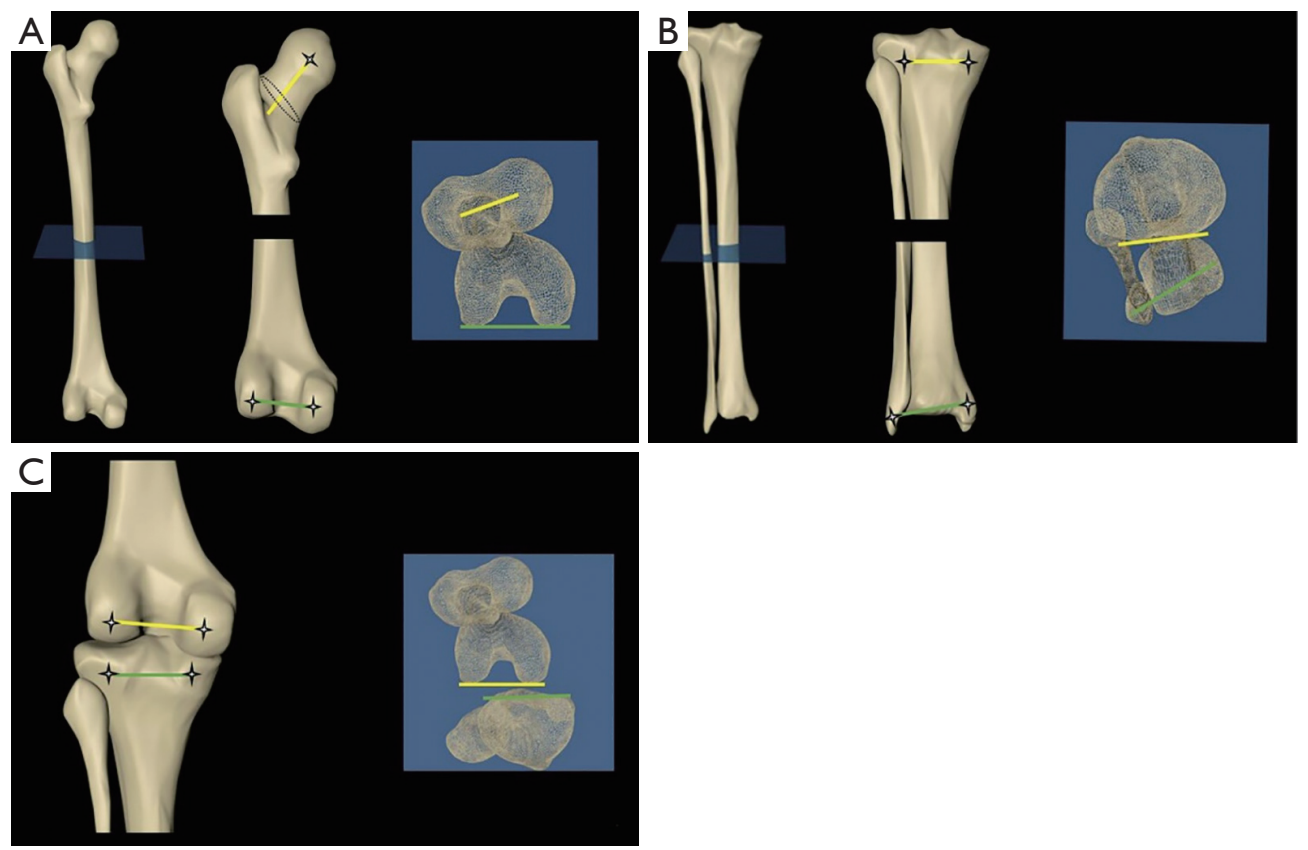

Figure 2 EOS measurement of femoral torsion, tibial torsion and femorotibial rotation. The yellow and green lines are projected on a plane that is orthogonal to the mechanical axis. The acute angle formed by the two projected lines was measured. (A) EOS measurement of femoral torsion; (B) EOS measurement of tibial torsion; (C) EOS measurement of femorotibial rotation. EOS, EOS imaging system (EOS imaging). 

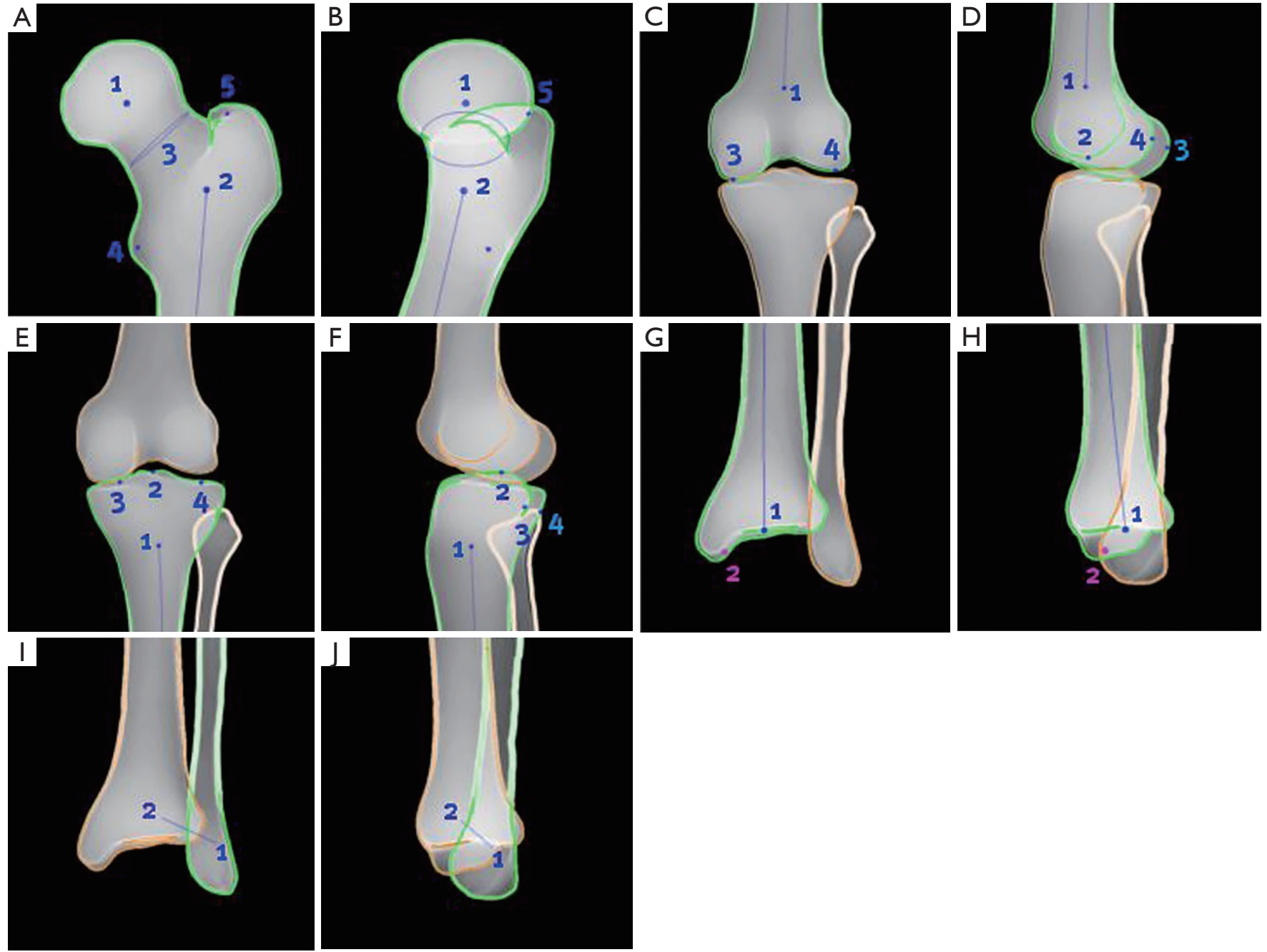

Figure 3 Three-dimensional model based on biplanar radiographs. (A-J) Computer model (green) is adapted semiautomatically to osseous contour of femur and tibia on anteroposterior (A,C,E,G,I) and lateral (B,D,F,H,J) radiographs. Three-dimensional models are built on the basis of fitting process. Fitting process is facilitated with standardized points on osseous contour that can easily be adjusted by dragging and dropping. The key points that are crucial for torsion measurements are greater trochanter at the hip (A5,B5), the posterior contours of the femoral condyles (C3,D3: medial condyle; C4,D4: lateral condyle), the posterior contour of the medial and lateral aspects of the tibial head (E3,F3: medial aspect; E4,F4: lateral aspect), and the malleoli of the ankle joint (G2,H2: medial malleoli; I1,J1: lateral malleoli).

Then the most posterior point of the medial and lateral condyle and the lowest point of the greater trochanter, located in the middle between the medial and lateral condyle, were connected by a horizontal line (Figure 4B, 2). The femoral torsion is defined as the acute angle formed by the horizontal line (Figure 4B,2) and the line that combined with the femoral head rotation center and the midpoint of the narrowest femoral neck (Figure 4B, 1). If the femoral neck is anteverted, then the angle is positive; the retroverted neck is corresponded to a negative angle.
The 3D-CT images were reconstructed to measure the tibial torsion angle. The proximal reference axis was the posterior condylar axis that passed through the two most posterior points on the tibial plateau (Figure $4 C, 1$ ). The transmalleolar axis that connected to the most protrusions of the medial and lateral malleolus (Figure 4C, 2) was determined as the distal reference axis. The two lines were angulated to form tibial torsion. If the malleolus rotates externally relative to the tibia, then the angle is positive. The femorotibial rotation was the angle formed by the 
A

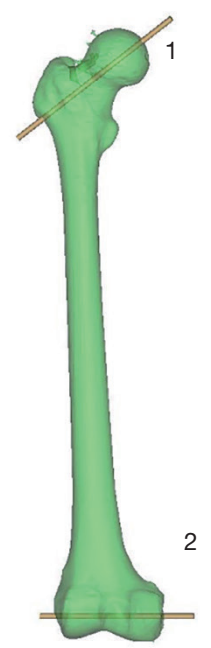

C

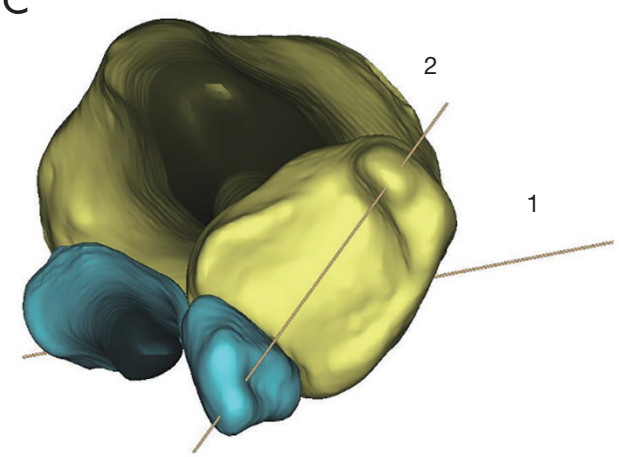

B

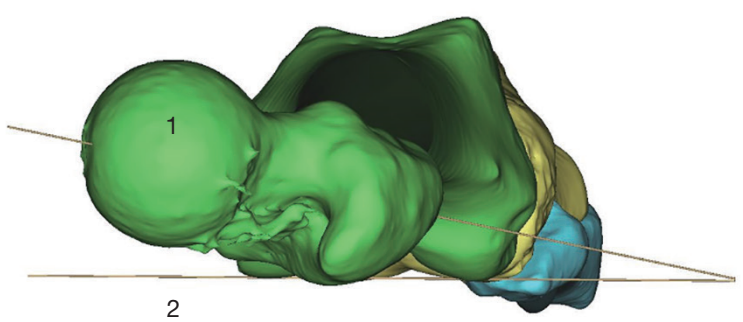

$\mathrm{D}$

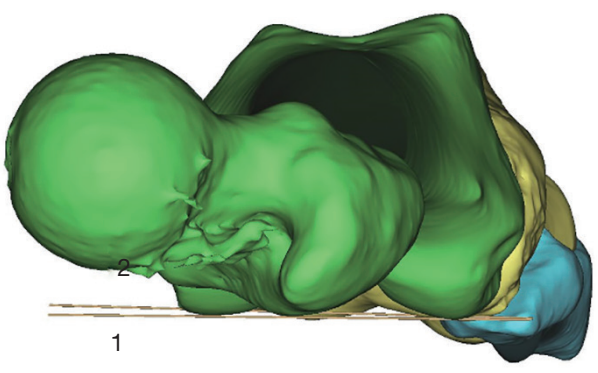

Figure 4 3D-CT reconstruction of lower extremity and the reference axis determination. (A) The transparent 3D reconstruction model of the femur on the frontal view; (B) femoral torsion measurement; (C) tibial torsion measurement on the caudo-cranial view of 3D-CT; (D) femorotibial rotation measurement. 3D-CT, three-dimensional computed tomography.

horizontal line of femoral condyle (Figure $4 D, 1)$ and proximal reference axis of tibial plateau (Figure $4 D, 2$ ). If tibia rotates externally relative to femur, then angle is positive. Yan, an orthopaedist with three-year experience, performed all measurements.

\section{Statistical analysis}

Statistical analysis was performed using MedCalc software (version 15.2.2, MedCalc). Bland-Altman plots were used to assess the differences in the femoral and tibial torsion measurements between the CT-based 3D modeling and EOS-based 3D modeling results $(17,18)$. Intrareader agreement was assessed by the intraclass correlation coefficient (ICC) with two-way model and consistency type. The ICC was calculated with SPSS statistical software (version 16.0, SPSS).

\section{Results}

Good agreement was exhibited between the measurements acquired by $3 \mathrm{D}$ models based on EOS imaging and those based on the three-dimensional CT reconstruction method. For the EOS imaging measurement, the mean measurement results were $22.1^{\circ}$ for femoral torsion, $30.2^{\circ}$ for tibial torsion and $4.9^{\circ}$ for femorotibial torsion. The corresponding values for the three-dimensional CT reconstruction measurement were $15.9^{\circ}, 29.1^{\circ}$, and $4.5^{\circ}$ (Table 1).

Bland-Altman plots showed that the mean differences between CT and EOS measurements were $3^{\circ}$ (range, $-9^{\circ}$ to $4^{\circ}$ ) for the femoral torsion, $0^{\circ}$ (range, $-6^{\circ}$ to $6^{\circ}$ ) for the tibial torsion and $0^{\circ}$ (range, $-4^{\circ}$ to $5^{\circ}$ ) for the femorotibial torsion. The variability in measurement results was consistent throughout the graph, and the scatter showed no tendency to increase as the mean of the EOS and CT 


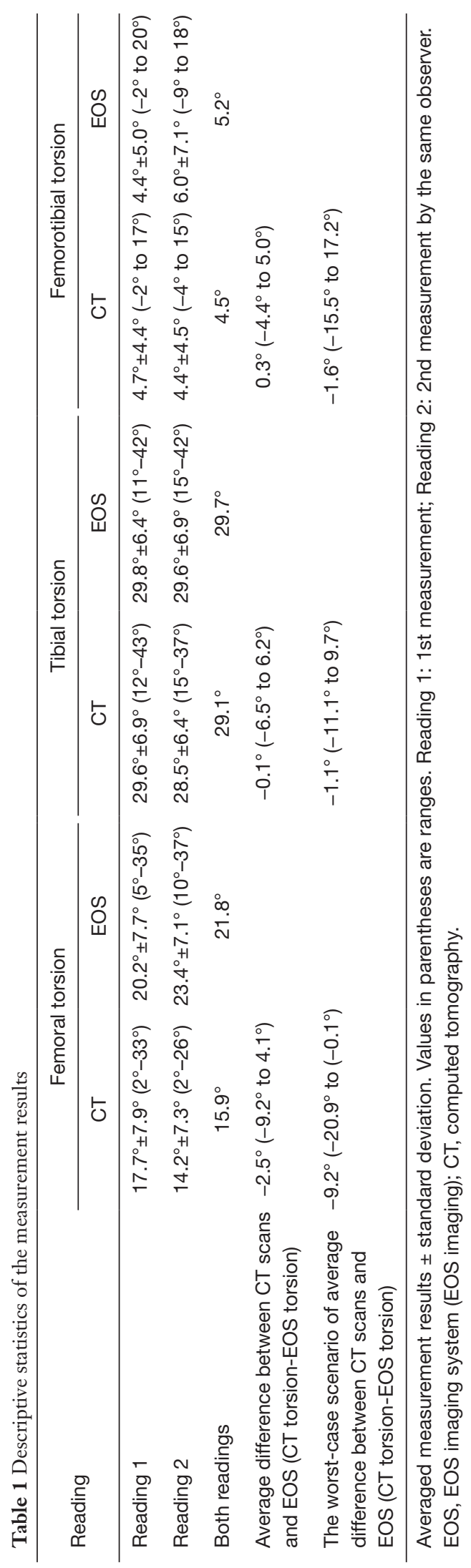

measurements increased. Excluding the results for one measurement of femoral torsion, one measurement of tibial torsion and one measurement of femorotibial torsion, all measurements of EOS imaging were within the $95 \%$ limits of agreement (Figure 5).

Intrareader agreement was statistically significant $(\mathrm{P}<0.001)$ for all measurement results. For the threedimensional CT reconstruction method, ICC was 0.950 (95\% CI, 0.902-0.975) for femoral measurement, 0.927 (95\% CI, 0.857-0.963) for tibial measurement and 0.889 (95\% CI, 0.782-0.943) for femorotibial measurement. The measurement results obtained with $3 \mathrm{D}$ models based on EOS imaging were similarly characterized and demonstrated high intrareader agreement, with an ICC of 0.920 (95\% CI, 0.843-0.959) for femoral torsion, 0.920 (95\% CI, 0.835-0.961) for tibial torsion and an even higher ICC of 0.918 (95\% CI, 0.827-0.961) for femorotibial torsion when compared to the measurements obtained with CT (Table 2).

\section{Discussion}

A previous study showed that the performance of femoral and tibial torsion measurements acquired with EOS imaging were comparable to those of two-dimensional CT scans in patients with knee osteoarthritis who were scheduled to receive arthroplasty (15). Three-dimensional CT reconstruction enables more accurate measurements of femoral and tibial torsion than two-dimensional CT scans (19), without being influenced by femoral or tibial positioning and the determination of femoral neck axis. The accuracy of the measurement results performed in patients with knee osteoarthritis may be influenced by the existence of osteophytes and lower limb deformities, which are typically absent in normal adults. Therefore, comparing the effectiveness of EOS imaging based and 3D CT reconstruction based technique in measuring femoral, tibial and femorotibial torsion in adults was more reliable.

In our study, very good agreement between the two methods was observed. The variability between the two methods showed no tendency to increase (or decrease) as the mean of CT and EOS measurements increased, as displayed by the Bland-Altman plots. The mean difference between the two methods was $3^{\circ}$ for the femoral measurements, $0^{\circ}$ for the tibial measurements and $0^{\circ}$ for the femorotibial measurements, indicating good consistency between the two methods for measuring femoral, tibial and femorotibial torsion. The intrareader agreement of the femoral, tibial 

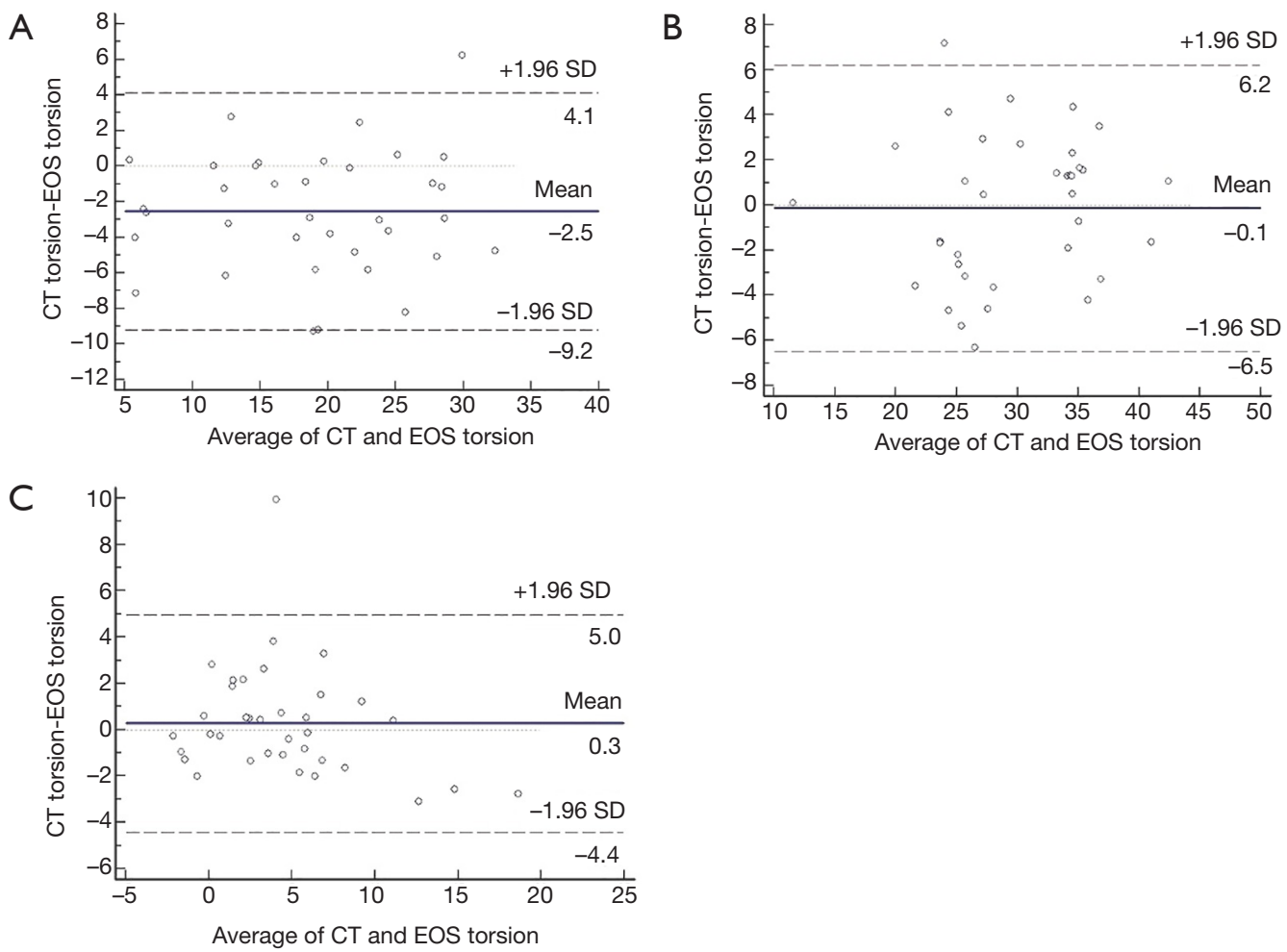

Figure 5 Bland-Altman plots of femoral (A), tibial (B) and femorotibial (C) torsion measurements. EOS, EOS imaging system (EOS imaging).

Table 2 Intrareader agreement (ICC) of torsion measurements using the EOS system and CT

\begin{tabular}{lcccc}
\hline \multirow{2}{*}{ Variable } & \multicolumn{2}{c}{ EOS } & & \multicolumn{1}{c}{ CT } \\
\cline { 2 - 5 } & ICC & $95 \% \mathrm{Cl}$ & $\mathrm{ICC}$ & $95 \% \mathrm{Cl}$ \\
\hline Femoral torsion & 0.920 & $0.843-0.959$ & 0.950 & $0.902-0.975$ \\
Tibial torsion & 0.920 & $0.835-0.961$ & 0.927 & $0.857-0.963$ \\
Femorotibial torsion & 0.918 & $0.827-0.961$ & 0.889 & $0.782-0.943$ \\
\hline
\end{tabular}

ICC, intraclass correlation coefficient; 95\% Cl, confidence interval; EOS, EOS imaging system (EOS imaging); CT, computed tomography.

and femorotibial torsion measurements seemed similar for the two methods, with ICC values equal to or greater than 0.9 , except for that of the ICC of the femorotibial torsion measured by CT, indicating a very strong correlation (20). The torsion measurements are important for preoperative planning, as described above. For example, for total knee arthroplasty, patient-specific cutting blocks are produced for accurate $3 \mathrm{D}$ preoperative planning, and these are mainly based on previous CT data. However, the feasibility of obtaining the necessary data for producing these blocks from EOS imaging could potentially be supported by our results.
The accurate recognition of landmarks required for EOS 3D model reconstruction depends on ensuring the correct standing position before image acquisition. This was achieved by placing one foot (usually the left) slightly anterior to the other. Otherwise, superimposed knees can cause difficulty in identifying the posterior edge of the femoral condyle and the tibial plateau on the lateral image.

For one volunteer, the results of the femoral, tibial and femorotibial torsion measurements obtained by EOS imaging exceeded the $95 \%$ limits of agreement. The superimposition of the patient's legs (Figure 6) caused difficulties in the identification of the anatomical landmarks. 


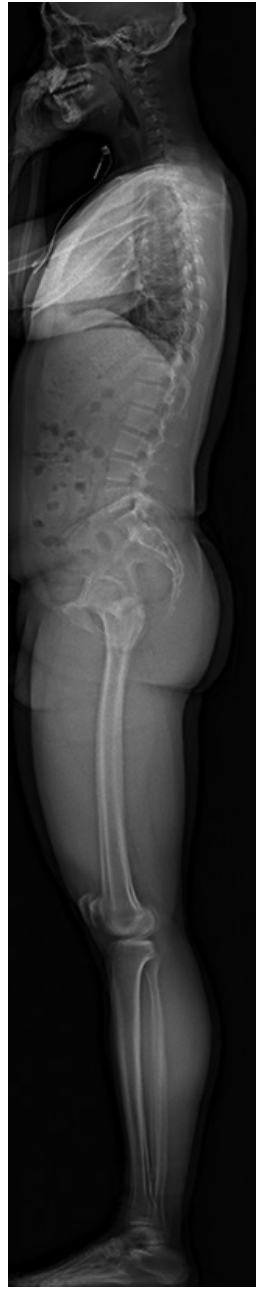

Figure 6 lateral radiograph of EOS imaging with two legs superimposed. EOS, EOS imaging system (EOS imaging).

Therefore, substantial errors can be produced and the measurements showed poor agreement. As a consequence, a second image acquisition was demanded when the superimposed legs on the EOS image occurred in the clinic.

The collection of $3 \mathrm{D}$ data based on biplanar radiographs is a major advantage of the EOS system, thus providing a new choice for femoral and tibial torsion measurement instead of preoperative radiographs and CT scans. The EOS system is especially useful for patients with multiple lower-extremity abnormalities, particularly when multiple deformities exist simultaneously (21). Because the irradiation exposure of EOS is considerably lower than that of X-ray and CT $(22,23)$, torsion measurements based on EOS imaging are beneficial for children and young adults (24), particularly for patients requiring extensive orthopedic imaging studies. CT scanning creates considerably higher radiation doses, which largely depends on the scanning area covered by the scanner. Furthermore, more measurements can be simultaneously acquired with EOS imaging, including the mechanical axis of the lower limb and femoral offset (25), as the radiographs are obtained under upright weight-bearing positions. The $3 \mathrm{D}$ analysis process is composed of a semiautomatic software-guided procedure that can be operated more easily than $3 \mathrm{D} C \mathrm{CT}$ analysis completed with Mimics software.

Several deficiencies also exist in the EOS system. Standing without moving for approximately $15 \mathrm{~s}$ is the basic requirement for acquiring biplanar lower limb radiographs (22). Therefore, patients who are unsteady in the standing position are not candidates for EOS imaging of the lower extremities. Furthermore, it can be difficult to identify anatomical landmarks in patients with advanced hip or knee osteoarthritis due to prominent deformities or marked osteophyte formation (16). This limitation also influences torsion measurement with EOS and CT. The intraclass diversity within EOS measurements can also be caused by the process of identifying the landmarks manually. Additionally, the benefits to the patients, including lower radiation dose and $3 \mathrm{D}$ modeling analysis, must be justified for the expenditure of approximately 1 million dollars on the introduction of an EOS system, which would increase costs in the medical institutions of many countries.

Several limitations also exist in this study. Sample size and selection bias may be sources of error, as only 18 volunteers were included in this study. The sample size may be restricted by the fact that X-rays are harmful to the human body. Furthermore, we performed only two readings as a limitation to the assessment of the intrareader agreement. For further verification of the consistency between EOS imaging and three-dimensional CT reconstruction, future studies are needed to compare different populations of patients, including patients with knee or hip osteoarthritis or those who have had total hip arthroplasty, taking into account interreader agreement.

\section{Conclusions}

There was good agreement between EOS imaging based and $3 \mathrm{D}$ CT reconstruction based technique in measuring femoral, tibial and femorotibial torsion; and good reliability and reproducibility of EOS Imaging in measuring femoral, 
tibial and femorotibial torsion was also verified.

\section{Acknowledgments}

We thank the volunteers who participated in this study. Funding: This work was supported by National Key R\&D Program of China (2018YFC1105904), Natural Science Foundation of Jiangsu Province, China (BK20180127), Key Program of NSFC (81730067), Excellent Young Scholars NSFC (81622033), National Science Foundation of China (81802196), Social Development Project of Jiangsu Provincial Science and Technology Department (BE2016609).

\section{Footnote}

Conflicts of Interest: The authors have no conflicts of interest to declare.

Etbical Statement: The authors are accountable for all aspects of the work in ensuring that questions related to the accuracy or integrity of any part of the work are appropriately investigated and resolved. The study received approval from the institutional ethics committee and obtained informed consent.

\section{References}

1. Fouilleron N, Marchetti E, Autissier G, et al. Proximal tibial derotation osteotomy for torsional tibial deformities generating patello-femoral disorders. Orthop Traumatol Surg Res 2010;96:785-92.

2. Krengel WF 3rd, Staheli LT. Tibial rotational osteotomy for idiopathic torsion. A comparison of the proximal and distal osteotomy levels. Clin Orthop Relat Res 1992;(283):285-9.

3. Pirpiris M, Trivett A, Baker R, et al. Femoral derotation osteotomy in spastic diplegia. Proximal or distal? J Bone Joint Surg Br 2003;85:265-72.

4. Tonnis D, Heinecke A. Acetabular and femoral anteversion: relationship with osteoarthritis of the hip. J Bone Joint Surg Am 1999;81:1747-70.

5. Hernandez RJ, Tachdjian MO, Poznanski AK, et al. CT determination of femoral torsion. AJR Am J Roentgenol 1981;137:97-101.

6. Liodakis E, Doxastaki I, Chu K, et al. Reliability of the assessment of lower limb torsion using computed tomography: analysis of five different techniques. Skeletal
Radiol 2012;41:305-11.

7. Morvan G, Testard S, Busson J, et al. Computed tomographic measurement of the angle of torsion of the femoral neck. Experimental reliability study on dry bone. Rev Chir Orthop Reparatrice Appar Mot 1987;73:511-6.

8. Sugano N, Noble PC, Kamaric E. A comparison of alternative methods of measuring femoral anteversion. J Comput Assist Tomogr 1998;22:610-4.

9. Morvan G, Guerini H, Carre G, et al. Femoral Torsion: Impact of Femur Position on CT and Stereoradiography Measurements. AJR Am J Roentgenol 2017;209:W93-9.

10. Jia J, Li L, Zhang L, et al. Three dimensional-CT evaluation of femoral neck anteversion, acetabular anteversion and combined anteversion in unilateral DDH in an early walking age group. Int Orthop 2012;36:119-24.

11. Shin SY, Yoon CH, Lee ES, et al. The availability of radiological measurement of tibial torsion: threedimensional computed tomography reconstruction. Ann Rehabil Med 2011;35:673-9.

12. Dubousset J, Charpak G, Skalli W, et al. EOS stereoradiography system: whole-body simultaneous anteroposterior and lateral radiographs with very low radiation dose. Rev Chir Orthop Reparatrice Appar Mot 2007;93:141-3.

13. Lazennec JY, Rangel A, Baudoin A, et al. The EOS imaging system for understanding a patellofemoral disorder following THR. Orthop Traumatol Surg Res 2011;97:98-101.

14. Chaibi Y, Cresson T, Aubert B, et al. Fast 3D reconstruction of the lower limb using a parametric model and statistical inferences and clinical measurements calculation from biplanar X-rays. Comput Methods Biomech Biomed Engin 2012;15:457-66.

15. Buck FM, Guggenberger R, Koch PP, et al. Femoral and tibial torsion measurements with 3D models based on lowdose biplanar radiographs in comparison with standard CT measurements. AJR Am J Roentgenol 2012;199:W607-12.

16. Folinais D, Thelen P, Delin C, et al. Measuring femoral and rotational alignment: EOS system versus computed tomography. Orthop Traumatol Surg Res 2013;99:509-16.

17. Bland JM, Altman DG. Statistical methods for assessing agreement between two methods of clinical measurement. Lancet 1986;1:307-10.

18. Mantha S, Roizen MF, Fleisher LA, et al. Comparing methods of clinical measurement: reporting standards for bland and altman analysis. Anesth Analg 2000;90:593-602.

19. Kaiser P, Attal R, Kammerer M, et al. Significant differences in femoral torsion values depending on the 
CT measurement technique. Arch Orthop Trauma Surg 2016;136:1259-64.

20. Sundmacher L, Gotz N, Vogt V. Statistical methods for research on regional health-care services. Bundesgesundheitsblatt Gesundheitsforschung Gesundheitsschutz 2014;57:174-9.

21. Thelen P, Delin C, Folinais D, et al. Evaluation of a new low-dose biplanar system to assess lower-limb alignment in 3D: a phantom study. Skeletal Radiol 2012;41:1287-93.

22. Dubousset J, Charpak G, Dorion I, et al. A new 2D and 3D imaging approach to musculoskeletal physiology and pathology with low-dose radiation and the standing position: the EOS system. Bull Acad Natl Med

Cite this article as: Yan W, Xu X, Xu Q, Yan W, Sun Z, Jiang Q, Shi D. Femoral and tibial torsion measurements based on EOS imaging compared to 3D CT reconstruction measurements. Ann Transl Med 2019;7(18):460. doi: 10.21037/atm.2019.08.49
2005;189:287-97; discussion 297-300.

23. Delin $\mathrm{C}$, Silvera $\mathrm{S}$, Bassinet $\mathrm{C}$, et al. Ionizing radiation doses during lower limb torsion and anteversion measurements by EOS stereoradiography and computed tomography. Eur J Radiol 2014;83:371-7.

24. Meyrignac O, Moreno R, Baunin C, et al. Low-dose biplanar radiography can be used in children and adolescents to accurately assess femoral and tibial torsion and greatly reduce irradiation. Eur Radiol 2015;25:1752-60.

25. Flecher X, Ollivier M, Argenson JN. Lower limb length and offset in total hip arthroplasty. Orthop Traumatol Surg Res 2016;102:S9-20. 